\title{
Welfare Assessment Tools in Zoos: From Theory to Practice
}

\author{
Narelle Jones ${ }^{1 *}$, Sally L Sherwen ${ }^{2,3}$, Rachel Robbins ${ }^{4}$, David J McLelland ${ }^{1,4}$ and Alexandra L. Whittaker ${ }^{1}$ \\ 1 School of Animal \& Veterinary Sciences, The University of Adelaide, Adelaide, SA 5371, Australia; \\ 2 Wildlife Conservation and Science, Zoos Victoria, Melbourne, VIC 3052, Australia \\ 3 The Animal Welfare Science Centre, The University of Melbourne, Melbourne, VIC 3052, Australia \\ 4 Zoos South Australia, Adelaide, SA 5000, Australia, dmclelland@zoossa.com.au \\ * Correspondence: a1629100@student.adelaide.edu.au
}

\begin{abstract}
Zoos are increasingly putting in place formalized animal welfare assessment programs to allow monitoring of welfare over time, as well as to aid in resource prioritization. These programs tend to rely on assessment tools that incorporate resource-based and observational animal-focused measures since it is rarely feasible to obtain measures of physiology in zoo-housed animals. A range of assessment tools are available which commonly have a basis in the Five Domains framework. A comprehensive review of the literature was conducted to bring together recent studies examining welfare assessment methods in zoo animals. A summary of these methods is provided with advantages and limitations of the approaches presented. We then highlight practical considerations with respect to implementation of these tools into practice, for example scoring schemes, weighting of criteria, and innate animal factors for consideration. It is concluded that there would be value in standardizing guidelines for development of welfare assessment tools since zoo accreditation bodies rarely prescribe these. There is also a need to develop taxon or species- specific assessment tools to inform welfare management.
\end{abstract}

Keywords: Zoo animal welfare; Five Domains; Validity; Animal-based; Resource-based; Scoring

\section{Introduction}

In recent years the welfare of animals held in zoos has been of increasing interest to the public, with an expectation that high standards of welfare are achieved [1,2]. It has been suggested that even the goal of species conservation, the primary objective for many zoos, may not justify the housing of animals in captive environments if high standards of animal welfare are not apparent [3]. Certainly, animal welfare has also become a higher priority within the zoo industry itself [4]. In response to this increasing concern, and to adhere with increasing regulatory oversight there has been a recent impetus on development and validation of methods to assess the welfare of zoo animals. Furthermore, formal assessment of welfare status has recently received increased focus as a component of zoo accreditation schemes, such as that of the Association of Zoos \& Aquariums [5], and Australasian Zoo and Aquarium Association [6] whose program is based around the Five Domains model for welfare assessment. Institutions within these associations are required to complete and maintain industry accreditation which require formal processes for ongoing review and enhancement of animal welfare. These accreditation processes should be evidence-based and informed by research in welfare science around influencing factors such as the individual animal's nutrition, environment, health, behavior and overall mental state (Five Domains) [3].

There are multiple methods used to assess animal welfare, including assessment of physiological, immunological, or behavioral responses elicited in response to an intervention or housing environment $[7,8]$. Whilst it is commonplace to combine methods to enable a holistic determination of welfare state, methods for assessment of zoo animals should be non-invasive and undemanding on resources. Behavior-based methods are 
therefore likely to be the most practical to apply. A further consideration is the balance between resource-based, and animal-based measures. The former relate to the animals' environment and are often referred to as 'inputs'. They may include determinations of space allowance, temperature or humidity, or appropriate food presentation and nutritional value. They are generally quantitative, highly repeatable across different observers, and easy to record. However, these measures may not be correlated with the actual affective state or condition of an animal [3,9]. A recent shift in the trend of welfare science has seen the supplementation of resource- based assessments with animal- based indicators $[9,10]$. Animal- based indicators directly measure a combination of physical, behavioral and physiological variables and also consider the varied response of individuals to the same provision of resources [3,9]. Animal-based indicators are considered superior since they provide direct information on animal affective state [11]. From a practical viewpoint, a combination of both resource- and animal based-indices is likely to provide a more holistic assessment of animal welfare, whilst being reasonably practical to implement [12].

A further challenge in assessing animal welfare is that it is now well established that consideration should be given to the promotion of positive mental states, as well as the assessment of both positive and negative states [13]. It should also be noted that the presence of negative metal states does not necessarily equate to poor welfare. For example, it has been suggested that many species may be required to overcome short term challenges which may incite temporary negative states, in order to experience fundamentally and lasting levels of good welfare [14]. Creating the opportunity for animals to experience positive states requires that animals have agency, and can therefore make choices about their activities and have a level of control over outcomes in their life [15]. This may even lead to a positive feed forward mechanism where animals with positive welfare are more likely to engage in activities that enhance their welfare $[16,17]$. Whilst this shift in focus is widely accepted, it is generally regarded as problematic since current assessment methods are relatively more extensive, and developed, for the assessment of negative emotions such as pain or fear, than they are for positive emotions. Furthermore, there has been less research attention directed towards identification of indicators of positive emotional state in some phyla, such as reptiles or amphibians, and even some mammalian species, in comparison to other more common domesticated species.

In this review we propose a 'how-to' guide to creating welfare assessment tools for zoological parks. This guidance is based on an overview of the various theoretical foundations that have been used to derive welfare assessment tools for zoo animals, together with discussion of the considerations necessary to turn these into practical and quantifiable instruments. The latter includes aspects such as scoring schemes, weighting of criteria, validity/reliability, and the split between animal and resource-based assessments, whilst considering factors such as seasonality, innate behaviors and an individual animal's exposure to training.

\section{Defining welfare and emotion}

There is no definitive definition for the term animal welfare; however, it is generally considered that 'animal welfare' comprises a physical and mental element, whilst ensuring opportunities for the animal to display a range of natural behaviors are provided [18]. Welfare is generally considered to be a long-term state, that is made up of the sum of experiences of an individual. These individual experiences are affective states, described in terms of their characteristics of valence and arousal. Affective states have been variously described as an animal's feelings, emotions or moods. They are commonly described in terms of their dimensions which comprise 1) direction of effect i.e. positive or negative (valence) and 2) arousal (level of activation) [7]. Individuals experiencing 
mostly positive affective states, such as reward, are said to have good welfare. Alternatively, animals that mostly experience negative affective states such as pain or fear, are described as having poor welfare [19]. As previously discussed, in spite of the availability of a wide variety of physiological and other biological methods for ascertainment of the nature of affective states [20], these are commonly invasive or impractical to implement in a zoo setting on a regular basis. Consequently, behavioral and resource-based measures predominate and are the focus of this review as a basis for performing institutional based welfare assessments of animals.

The use of behavioral indicators is a practical, non-invasive method of measuring affective states in zoo animals [10]. However, the difficulty lies in identifying and validating taxon or species- specific indicators and relating them to affect. It is generally regarded that for behavior to be a useful indicator, there is a need for animal caretakers to be familiar with the full range of normal behaviors for the species being assessed [21]. The behavior must also occur frequently enough for identification to be practical. For many species held in zoos there is limited literature on natural behavioral biology and thus, determining useful indicators can be a challenge [21]. Facial expressions, vocalizations, social interactions, and 'opportunity situations' such as inquisitive exploration and play are examples of behavioral indicators of good welfare [10,12] with strong potential for inclusion in zoo animal assessment tools due to their practicality and non-invasive nature. However, many of these have not been characterized in zoo species. Moreover, ideally there should be empirical evidence that the behavior relates to the affective valence of the animal, and the direction of that affect [22]. In the absence of this direct evidence, those creating assessment tools should consider how they can demonstrate the link between the behavior and affect by inductive reasoning [22].

It is important to note that whilst behavioral measures are practical and inexpensive indicators of welfare, caution should be taken when interpreting observations. Many species do not clearly express behavioral signs of poor welfare [10] and some have adapted methods to avoid displaying signs of pain or distress [23]. Other species may express behavioral indicators, but individuals may display varied responses to the same welfare compromise [21]. Furthermore, when behavior monitoring is incorporated into an assessment tool, the behavior should be fully defined, including the context in which it may be seen. A behavior that may occur in different contexts may then legitimately be included in different functional categories [22].

Hence, where possible, a combination of behavioral and practically obtainable physiological and biological or health markers should be considered when developing a welfare assessment tool [10]. Use of established, validated assessment frameworks will assist in achieving this

\section{Frameworks and protocols utilized for welfare assessment in zoo animals}

The five freedoms were first proposed by Brambell 1965, and are listed by the FAWC as: Freedom from hunger and thirst; Freedom from discomfort; Freedom from pain, injury and disease; Freedom from fear and distress, and; Freedom to express normal behavior [24]. These freedoms have been widely used to describe welfare [12] and are still used today, particularly in legislative documents [25].In recent years the development of the Five Domains Model by Mellor has addressed some of the deficiencies with the Five Freedoms model, paying greater consideration to positive experiences, and the mental state of an animal [26].

These frameworks have become the foundation for the development of a range of welfare assessment tools across multiple species of captive animals [27], including those held in zoos. Named models are described below with a brief overview of their use in relation to animals in zoos. 


\subsection{Five Domains model for animal welfare assessment}

The Five Domains model for animal welfare assessment references four physical domains; nutrition, environment, health and behavior. The fifth domain, mental state, enables the assessment of the animal's overall affective state resulting from the physical domains described [28]. In spite of the adoption of the Five Domains by the World Association of Zoos and Aquaria, and the Zoo and Aquarium Association of Australasia (ZAA) as the framework for welfare assessment and consequent accreditation under their schemes, there is little published literature on the practical implementation of this in zoological parks. These organizations have however suggested that the model is practical and readily adaptable to a range of species [12].

Perhaps the largest scale adoption of this framework published in relation to zoo animals was that carried out by Sherwen and colleagues [3], where zoo-wide use was trialled across 339 species in three zoos. In this study, the authors used the Five Domains model as a tool to conduct institutional -level assessment of welfare risk factors, as opposed to individual animal welfare monitoring. The former type of assessment may be better able to inform resource allocation, and have greater application in benchmarking [3]. The resulting process has value in highlighting areas of risk for suboptimal welfare, whilst simultaneously providing opportunities for improvement. However, the method may not be suitable for use as the sole process for animal welfare assessment. This is because the process derived was heavily focused on assessment of resources or inputs, and whilst provision of resources may translate to good welfare, this is not always a given. The incorporation of animal-based measures, being more direct measures of affective state [29], is recommended to augment this process. However, there is still a relative lack of information on indicators of affective state in a number of animals housed in zoos, particularly birds, reptiles and amphibians. Hence, supplementary processes or research may be required to gather these data to incorporate into the Five Domains-based tool [3].

\subsection{The European Welfare Quality® animal welfare assessment protocol}

The European Welfare Quality ${ }^{\circledR}(\mathrm{WQ})$ animal welfare assessment protocol for farm animals was originally designed to scientifically assess the welfare of production animals based on four principles, similar to those in the Five Domains model. The four principles, being good feeding, good housing, good health, and appropriate behavior, are defined by a set of 12 criteria deemed meaningful to the animals [30]. These criteria are allocated measurable indicators based on the opinion of expert consultants. An important aspect is the three-step approach to scoring where, 1) welfare measures, for example body condition score or space allowance, make up criteria scores, 2) criteria scores integrate into scoring for the four principles and 3) an overall assessment of the enterprise is made based on combining the principle scores [31]. However, the practicality of this protocol is significantly reduced due to the amount of time and resources required for implementation, largely as a result of the number of criteria [9]. However, further testing and validation of the tool may lessen this. For example, that there was inherent redundancy in production animals, with welfare classification being accounted for using only two resource-based measures [32,33].

Mononen et al (2012), in their adaptation of the protocol to farmed foxes and mink provide a useful discussion of the considerations for adaptation of the protocol to other species [31]. A key point is that whilst some welfare measures may be relevant for multiple species, sets of measures and how their evaluation feeds into criteria scores i.e. their weighting, is species- specific. Therefore, welfare indicators that are valid, feasible and reliable for the species of interest are first required. These may be derived from literature review or expert opinion, perhaps using Delphi techniques (see later). The protocol emphasizes the desirability of including as many animal-based measures, as opposed to resource-based measures, as possible [34]. Where this is not feasible there is recognition 
that it may be appropriate to use an input-based measure that shows a good correlation with an animal-based measure [34]. Welfare measures selected should also be independent of each other [35].

The first adaptation to animals in a zoo setting was a Cetacean Welfare Assessment process (C-Well), applied to bottlenose dolphins [36]. The measures were derived from published literature on dolphin health, behavior and physiology based on how these measures differentiated normal from abnormal states. Advice from marine mammal specialists, veterinarians and welfare scientists was incorporated resulting in measures that comprised those recorded whilst animals were working with a trainer, observations when not with a trainer, opportunistically-derived, and those obtainable from records or questioning of the curator. The tool developed was considered to be useful for assessment of individual welfare, chosen demographics and whole facilities, and was practical to implement, although in this first iteration it was suggested that it be performed by individuals trained in the methodology and metrics. The WQ has since been applied successfully to Dorcas gazelles [37], and proposed for the pygmy blue-tongue skink [9].

\subsection{Qualitative Behavior Assessment}

Qualitative Behavior Assessment (QBA) is a method of summarizing and evaluating an animal's behaviors and emotions in relation to their environment using descriptive terms such as 'calm', 'timid' or 'excited' [38]. It can be used retrospectively or applied to real-time welfare assessments. The method uses a comprehensive list of expressive terms, either generated by individual users when using the Free-Choice-Profiling methodology (FCP), or pre-determined from the literature and expert consultancy. As FCP requires the participation at least 10 observers and extensive data analysis [39], the latter is a more practical option for implementation in a zoo environment. QBA relies on the ability of keepers to observe subtle details of behavior, attitude, posture, and movement that may go undetected by other systematic behavioral data collection methods [10]. The literature shows good correlations between QBA and behavioral, physical and physiological measures of welfare and studies have found high levels of inter-rater reliability when performed correctly by experienced keepers [10,39]. It is worth noting that whilst QBA is a useful resource to indicate the positive aspects of an individual animal's welfare, it should not be used as a stand-alone assessment tool and cross validation with other measures is recommended [40].

In the context of animals in zoos, QBA has been used to develop a welfare assessment tool for captive elephants [41]. However, QBA was not used as the sole method of welfare assessment, and instead was combined with ethogram-type analysis of daytime and nighttime behaviors. This tool focused purely on behavior rather than incorporating measures of health and physical condition. Sixteen terms to describe demeanor were scored on a visual analog scale based on one-minute observation periods. The authors demonstrated that some QBA terms were able to be rated reliably by keepers and had an association with welfare state. Some terms were however unreliable, for example the term 'depressed', implying that further validation of descriptors is required, along with their validation against alternative measures of affective state. Whilst these species- specific methods of welfare assessment may offer a more detailed indication of welfare than some generic assessment tools, the vast number of species held in any one zoo makes it impractical to use species- specific tools for all. This notwithstanding, the generic nature of QBA along with the number of species for which its use has already been validated, would suggest that it could be adapted to a wide range of species housed in zoological parks. 


\subsection{Behavioral diversity as an indicator of welfare}

There has been a growing interest in recent years in the use of behavioral diversity as a measure of animal welfare. Behavioral diversity is defined as the number of behaviors, as well as the frequency of each behavior [42]. The assumption made is that when diversity is high, behavioral needs are being met, but when diversity is low an animal may be in poor welfare, for example they may show stereotypies or be lethargic [42]. The Shannon-Wiener Diversity Index (H-index) is perhaps the most used measure of diversity and provides an indication of whether an animal's time budget is mostly made up of a few behaviors or covers a range of behavioral categories [43]. The model closely aligns with the natural living model of welfare, in that animals in the wild display considerable behavioral diversity and hence good welfare in captivity would be assumed if animals showed similarly diverse behaviors [22]. Although, there are differences; using diversity indexes requires counting of individual behaviors whereas natural living compares behaviors that are similar between wild and captive counterparts. Therefore, if this assumption holds, a valid welfare assessment method would be comparison of time budgets between captive animals and their wild counterparts[44,45]. Alternately, ethogram comparisons between before and after environments, or conditions, would allow assessment of welfare status change [45]. However, there is a risk of arriving at incorrect assumptions about welfare. For example, the performance of behaviors associated with negative affective states may increase causing a change in diversity index, but welfare state may have declined [46]. For this reason, the inclusion of so-called 'negative' behaviors is needed to avoid misinterpretation.

In spite of these concerns, there is a growing attention to behavioral diversity as a valid positive indicator of welfare. Firstly, there is now an extensive body of evidence showing that management practices that are thought to improve welfare lead to increased diversity. For example, the impact of enrichment or animal training on greater behavior expression has been widely reported (see [42] for review). It is also promising that this change is occurring across orders and species and is not confined to mammals only. Secondly, there appears to be a relationship between diversity and physiological indicators of welfare, for example with corticosterone as a measure of HPA axis activity with the expected inverse relationships being found in cheetahs [47], and dolphins [48].

In practice the method probably has greatest application via use of an established diversity index prior to and following a husbandry change, rather than as a one-off assessment method. Monitoring of behaviors can be achieved through targeted focal animal sampling, with the use of technology to aid practicality, e.g. the use of the Zoo Monitor program. However, as described by Miller et al. 2020 [42], use of the method may require a shift in focus away from keeper-oriented husbandry programs with predictable scheduling to opportunity-based changes considering the behavioral outcomes observed. This paradigm shift would require whole-scale institutional endorsement and commitment to optimize success.

\subsection{Zoo specific welfare assessment programs}

In 2015, Kagan and colleagues proposed a universal animal welfare framework for zoos. The authors describe 4 main components that together ensure excellent animal well-being. These are: institutional philosophy and policy, programmatic structure and resources, execution and evaluation [15]. The framework recognizes that exemplary standards of care for animals does not necessarily lead to optimum welfare. Instead, there is a need for transparency, discussion, accountability and ongoing commitment and evaluation of policy. An important part of this is ensuring that resource allocation is adequate. Various factors play into the success of the proposed framework including staff training, an animal welfare communication process to include all stakeholders, welfare leadership, and a tiered evaluation process with internal and external welfare evaluations. The 
strength of this program is the holistic approach taken with the explicit recognition of a multitude of factors that impact on animal welfare and the assessment of it in an institutional context.

An Opportunities to Thrive program was created at San Diego Zoo Global (SDZG). This program specifically addresses the complexities of conservation breeding programs which need to consider optimising welfare whilst increasing the chances of successful reintroductions to the wild [49]. The program uses the Five Freedoms but transforms them from a focus on reducing negative indicators to the attainment of positive affect. For example, freedom from pain, injury and disease is transformed to the "opportunity" to achieve optimal health. Desired outputs or ways of assessing the criteria are defined, and these are targeted by management inputs that would help to achieve these, for instance the availability of health checks. In this way a structure is given to efforts to assess and improve welfare and the resulting program considers welfare outcomes in the context of management inputs. However, as with other assessment methods deriving suitable outputs can be challenging when there is a lack of knowledge about species-typical biology or behaviours.

\subsection{Delphi consensus methods}

Whilst not a welfare assessment technique, use of expert opinion can be a useful method for gathering information to feed into welfare assessment tools. For example, in cases of limited species literature on welfare indicators, the method can used to establish consensus by formalising a communication process to source this information from experts [50]. The technique usually comprises at least two rounds of questionnaires or interviews, with an iterative approach such that results from the first round are reconciled for the next round for futher questioning of participants [51]. The method has been used to identify welfare indicators in a range of species commonly housed in zoos including; elephants [52], tigers [53], and reptiles [54]. As the method relies solely on opinion (albeit expert), it is not possible to confirm linkage of indicators with valence of affect. However, confirmatory analysis of behaviours identified can be performed in later follow-on studies. Furthermore, the participatory nature of the method likely encourages communication and perhaps greater buy-in to the tools that arise by staff at zoological parks and their institutions.

\section{Important features of welfare assessment tools for zoos}

An individual animal's welfare state is multifactorial and as further understanding evolves, the importance of including animal- based measures in welfare assessments has been highlighted $[10,21]$. There is a consensus among researchers that in addition to ensuring all resource needs are met, positive affective states should be promoted. There are many factors to consider when developing a welfare assessment tool and determining how welfare should be measured and recorded can be a challenge $[10,12,55]$. The various conceptual frameworks have given rise to multiple tools but these are often developed for specific species or contexts and there is no 'one size fits all' [56]. Under captive conditions, such as those seen in zoos and aquaria, this variation may result in non-comparable data limiting the ability for knowledge- sharing and collaboration between organizations. Additionally, Fraser 2009 noted that the assessment of welfare using a tool created based on a single criterion may produce a poor welfare result when scored by a tool based on different criteria [57]. As a result, there is a requirement for standardized guidelines to developing zoo animal welfare assessment tools that are adaptable and flexible enough to suit various taxon or species in varying contexts, whilst still producing comparable datasets. The development of such guidelines would need to consider features such as validity and reliability and the split between animal and resource-based assessments. They would also need to consider factors such as seasonality, innate behaviors and individual animal's exposure to training. Consideration must also be given to 
social settings and maintaining a balance between providing breeding opportunities and species management. In addition, scoring schemes, the weighting of criteria and methods of determining criteria should be regarded.

\subsection{Tool features: validity, reliability, practicality}

A welfare assessment tool should be practical, reliable and simple enough to allow completion by observers with no specialist training, as well as being comprehensive enough to allow application to a variety of species. As previously mentioned, a combination of multiple measures should be used to determine an animal's welfare status. Prior to inclusion in an assessment tool, these measures need to be evaluated for practicality (how easily they can be observed), reliability (the consistency in which they can be assessed) and validity (the ability of the measurement to reflect the desired construct) [41]. It has been suggested by Yon and colleagues [38] that a fully validated assessment tool should evaluate the various types of validity and reliability against predetermined thresholds from the literature. Common types of reliability applied to welfare assessment include; inter- / intra- rater, test re-test reliability and internal consistency. Validity may refer to content, concurrent criterion or known groups criterion validity [41].

When developing a welfare assessment tool, the recommended frequency of implementation is another factor that must be considered in order for it to remain practical. If recommending daily use by keepers, the tool must be rapid and simple to complete. More thorough institutional assessment tools may need less frequent evaluations. If the data are to be collected over the long-term to chart trends, consideration to reliability across time incorporating aspects of inter and intra-observer variability is also important.

The training and species knowledge of keepers may also influence the type of measures and methods selected for inclusion in the assessment tool. Multiple studies have validated keeper assessments as a measure of welfare by correlating observations with other common physiological and behavioral indicators of welfare [58,59]. However, these types of assessment require observations by experienced care takers. There has also been suggestion that keepers tended to capture more subtle changes to welfare than researchers using retrospective analysis, although correlation between different methods of assessing welfare, such as behavioral observations versus general welfare indicators may not be strong [60]. Conversely, Wemelsfelder and colleagues found that when using QBA's, inter-rater reliability was high even when performed by observers with no previous experience with the species [38,61,62]. Behavioral measures, once cross-validated, are becoming increasingly used in welfare assessments due to their practicality and noninvasive nature [10]. When selecting behaviors for inclusion, thought must be given to how the behavior of the species may influence assessment. When evaluating less expressive species, care needs to be taken to select measures that will give a clear representation of the animal's welfare state. The same applies for species that may display seasonal differences in behavior, hibernate during certain periods of the year, or nocturnal species that may display different behaviors during the night when keepers are absent. Validating measures of welfare can be challenging in a zoo environment due to the often-small sample sizes and lack of literature on natural behavioral biology for many species [12]. However, there are numerous ways in which criteria can be selected for inclusion. A common approach includes a combination of reviewing the existing literature, gaining opinions through expert consultancy and modifying previously established and validated assessment tools. When developing a novel welfare assessment tool for captive elephants, Yon and colleagues conducted a review of both peer and non-peer reviewed literature and consulted stakeholder focus groups to identify novel measures for inclusion [41]. In their 2018 Assessment of Welfare in Zoo Animals, Wolfensohn and colleagues recommended the use of a targeted version of the Animal Welfare Assessment Grid (AWAG) to create a 'gold standard' welfare improvement benchmark for zoos over 
time [12]. AWAG is an easily adapted tool that has been previously validated in primates [63], and gorillas [60] and successfully adapted for use in zoos to highlight welfare impacts [64]. In addition, the AWAG system has been trialed on zoo collections of giraffe, scimitar horned oryx and numerous large felids [12].

\section{Factors for consideration in development of welfare assessment tools}

\subsection{Animal developmental stage and experience}

Animals of different ages may display varied responses to the same situation or provision of resources. This is likely representative of the situation having varied impacts on welfare of, animals at differing stages of physiological and neurological development, rather than for different ages groups [65].

The presence of abnormal behaviours are often included as a measure of welfare in behavioral-based assessment tools, however caution should be taken. Whilst the presence of steroetypic behaviours may indeed indicate compromised animal welfare, these behaviors have also been shown to improve welfare when performed during stressful situations, and may persist in the absence of welfare compromise [14]

The provision of enrichment has been found to significantly reduce the amount of time animals spend performing abnormal behaviours, such as engaging in stereotypic behaviour [66-68] A 2008 study explored the influence of age on the use of potential enrichment objects [in pigs] and found that time spent inactive varied between age groups; although animals of all ages used enrichment items when provided, younger animals displayed longer latency to approach novel objects and less object directed behaviour than their older counterparts [69]. Similarly in zoo animals, age was found to be an important determinant of responsiveness to enrichment objects in giant pandas (Ailuropoda melanoleuca) [68]; chimpanzees (Pan troglodytes) [70]; walrus (Odobenus rosmarus divergens) [71] and bottle nose dolphins (Tursiops truncatus) [72] . Thus, the age of individuals being observed should be considered when performing welfare assessments.

The relationship between captive animals and humans has been identified as a key contributing factor to the way in which animals interact with their environment [73]. An animal's level of exposure to training may act as a modifier of behaviour in that the individual may become more confident when interacting with humans. It is inferred that an increase in positive human- animal interactions (HAR) translates to an improved welfare state [73]. Training has been shown to reduce the occurrence of stereotypic behaviours in chimpanzees [74], and there is evidence to suggest participation in research tasks or training may itself be a source of enrichment for zoo animals [75-77]. Greco and colleagues (2016) reported that across 67 zoos in North America, the amount of time animals spent interacting with keepers (including training) of both African and Asian elephants increased with each year of age [78]. If this holds true across species, it can be assumed that older animals will have had greater exposure to training. Animals exposed to training may display a varied response to enrichment provision and reaction to keepers when compared to their lesser managed counterparts [78]. With behavioural interactions with humans being described in the most recent iteration of the Five Domains Model for welfare assessment [79], the use of HARs as a measure of welfare are likely to increase. Given the information above, it is important that the effects of age and an individual's 
exposure to training are considered when undertaking behavioural based welfare assessments.

\subsection{Seasonality/ Innate behaviours}

Significant variations in behaviour of zoo animals based on season and time of day are documented in the literature. This seasonality is evident in captive species including African elephants (Loxodonta africana) [80], Grizzly bears (Ursus Arctos Horribilis) [66], Rothschild's giraffes (Giraffa camelopardalis rothschildi) [81] and ring-tailed lemurs (Lemur catta) [82], highlighting the need for caution when using behavioural measures as indicators of welfare. For example, in some species, inactivity may be observed more frequently during winter to reflect innate hibernation behaviours [66] where other species may become more active [83]. Some animals might spend less time feeding and more time lying down during the warmer months [80]. Many species, including ruminants such as giraffe, show seasonal reproductive patterns [84] which may influence the frequency and type of social interactions observed. Thus, it is important that assessments are comprehensive enough to account for seasonal variability, and that evaluations of such behaviours do not produce a misleading negative partial welfare score. For this reason, there is increasing merit in the inclusion of keeper records in welfare assessments as caretakers are often most familiar with the individual temperaments, personalities and behaviours of the animals in their care [8].

\subsection{Scoring systems}

In order for an assessment tool to be valuable it must be able to record changes in welfare status over time. This is done by generating an objective welfare score which can be used for comparison in future evaluations. These scores are also used by accreditation schemes to determine if an organisation is meeting welfare requirements. Numerical scales are most common as they allow for ease of data analysis and comparison between datasets. Sherwen and colleagues implemented a 0-2 scale (where 0 represents high risk, 1 represents a moderate risk and 2 represents no observable risk) when developing their Animal Welfare Risk Assessment Process for Zoos [59]. This system was also used in a modified version of the Welfare Quality ${ }^{\circledR}$ Protocol to assess welfare in the Pygmy Blue-Tongue Skink [7]. Likert scales are also commonly utilised when evaluating behavioural indicators. When asking observers to score daytime behavioural frequencies in captive elephants, Yon and colleagues provided a variety of Likert scales with responses ranging from 'never' to 'more than once per day' where appropriate, and utilised various numbers of response options based on the expected frequency of that behaviour [38]. When combining results of welfare measures to gain an overall assessment score, there are a number of ways in which criteria may be weighted[85]. Delphi surveys or similar methods of expert consultancy, such as those employed by Veasey 2020 in an attempt to identify the psychological priorities of captive elephants, are an informal method of aggregating results to derive weighting based on validity, practicality and expected frequencies [48]. Alternatively, precise calculations can be used; the sum (or mean) of scores is commonly used since the concept can be easily 
grasped by a wide audience. In this method, all indicators are first converted to an ideally unified numerical scale (i.e., 0-2 scale used by Sherwen and colleagues [3]) before weightings are assigned. Measures that are considered to be less valid or impractical are assigned lower weightings, with higher weightings being assigned to those most likely to have an impact on the animals' welfare [35]. It is important to note that if a unified numerical scale is not implemented, the scale and intervals between levels for each measure should be illustrated to avoid confusion or misrepresentation. By combining the weighted sum of the scores an overall welfare score can be calculated. There are however some limitations to the use of this method. Scales and intervals between levels are often assumed not illustrated resulting in confusing or misleading results; the nature of the method allows for full compensation i.e a major welfare compromise may be neutralised by multiple minor advantage; and the method cannot favour situations of compromise. see [35] for full description. Scores may also be derived relative to a standard comparator. For example, in Brouwers and Duchateau's 2021 study using the AWAG for assessing gorilla welfare, indicators were scored relative to a healthy individual of the same sex and age, on a ten-point sclae with one as the optimum. In this study, an interesting visual method of presenting scores was used by plotting average scores in the four selected domains of physical, procedural, environmental and psychological o a radar plot. This allows calculation of a Cumulative Welfare Assessment Score (CWAS) based on the surface area of this chart. As a result the CWAS increases exponentially, rather than linearly when different classes are affected. This aids in capture of long-term trends in welfare [60].Having standardised scoring systems for zoos and aquaria would allow for ease of data comparison between organisations, allowing zoo managers to create a 'gold standard' to which all organisations should strive to achieve.

\section{Conclusions}

The focus of this review was to review the basis for welfare assessment frameworks that are in use or have been trialed in zoos with a focus on the foundation for the criteria derived, and to explore the method of deriving a practical and useable tool based on these foundational principles. With increasing interest from both the public and from within institutions themselves to continually improve the welfare of animals held in zoos, the use of tools to make evidence-based assessments of zoo animal welfare is crucial. Details of how to accomplish this are not always specified by accreditors and thus, the establishment of standardized guidelines for developing zoo animal welfare assessment tools would be beneficial for data comparison and sharing. The need to establish taxon or species- specific assessment tools across all taxonomic groups is also necessary to inform welfare management strategies.

\section{Author Contributions:}

Conceptualization, N.J, A.L.W., D.J.M., methodology, N.J, A.L.W., writing-original draft preparation, N.J, A.L.W., S.L.S.; writing - review and editing, N.J, A.L.W., S.L.S.; R.R, D.J.M.; supervision, D.J.M., S.L.S., R.R., A.L.W. All authors have read and agreed to the published version of the manuscript."

Funding: This research received no external funding.

Institutional Review Board Statement: Not applicable.

Informed Consent Statement: Not applicable.

Conflicts of Interest: The authors declare no conflict of interest. 


\section{References}

1. Phillips, C.; Izmirli, S.; Aldavood, S.; Alonso, M.; Choe, B.; Hanlon, A.; Handziska, A.; Illmann, G.; Keeling, L.; Kennedy, M. Students' attitudes to animal welfare and rights in europe and asia. Animal Welfare-The UFAW Journal 2012, $21,87$.

2. Coleman, G. Public animal welfare discussions and outlooks in australia. Animal Frontiers 2018, 8, 14-19.

3. Sherwen, S.; Hemsworth, L.; Beausoleil, N.; Embury, A.; Mellor, D. An animal welfare risk assessment process for zoos. Animals 2018, 8, 130.

4. $\quad$ Maple, T.L.; Perdue, B.M. Zoo animal welfare. Springer: 2013.

5. About AZA Accreditation I Association of Zoos \& Aquariums. (2021). Available online: https://www.aza.org/what-isaccreditation (accessed 28 April 2021).

6. Zoo Aquarium Association Australasia. Home. (2021). Retrieved 28 April 2021, Available online: https://www.zooaquarium.org.au/public/Home/Public/Default.aspx?hkey=72fe7386-d3c1-4f5a-9dc0-d7b565124e04 (accessed 28 ${ }^{\text {th }}$ April 2021).

7. Whittaker, A.L.; Marsh, L.E. The role of behavioural assessment in determining 'positive' affective states in animals. $C A B$ Reviews: Perspectives in Agriculture, Veterinary Science, Nutrition and Natural Resources 2019, 14, 1-13.

8. Broom, D. Animal welfare: Concepts and measurement. Journal of Animal Science 1991, 69, 4167-4175.

9. Benn, A.; McLelland, D.; Whittaker, A. A review of welfare assessment methods in reptiles, and preliminary application of the welfare quality ${ }^{\circledR}$ protocol to the pygmy blue-tongue skink, tiliqua adelaidensis, using animal-based measures. Animals 2019, 9, 27.

10. Whitham, J.C.; Wielebnowski, N. New directions for zoo animal welfare science. Applied Animal Behaviour Science 2013, 147, 247-260.

11. Manteca, X.; Temple, D.; Salas, M. Animal-based indicators to assess welfare in zoo animals. CAB Reviews: Perspectives in Agriculture, Veterinary Science, Nutrition and Natural Resources 2016, 11.

12. Wolfensohn, S.; Shotton, J.; Bowley, H.; Davies, S.; Thompson, S.; Justice, W. Assessment of welfare in zoo animals: Towards optimum quality of life. Animals 2018, 8, 110 .

13. Boissy, A.; Manteuffel, G.; Jensen, M.B.; Moe, R.O.; Spruijt, B.; Keeling, L.J.; Winckler, C.; Forkman, B.; Dimitrov, I.; Langbein, J., et al. Assessment of positive emotions in animals to improve their welfare. Physiolgy E Behavior 2007, 92, 375-397.

14. Veasey, J.S. In pursuit of peak animal welfare; the need to prioritize the meaningful over the measurable. Zoo Biology 2017, $36,413-425$.

15. Kagan, R.; Carter, S.; Allard, S. A universal animal welfare framework for zoos. Journal of Applied Animal Welfare Science 2015, 18, S1-S10.

16. Franks, B.; Champagne, F.A.; Higgins, E.T. How enrichment affects exploration trade-offs in rats: Implications for welfare and well-being. PLOS ONE 2013, 8, e83578.

17. Spinka, M., Wemelsfelder, F., Appleby, MC. (Ed.), Mench, JA. (Ed.), Olsson, A. (Ed.), \& Hughes, BO. (Ed.) (2011). Environmental challenge and animal agency. In MC. Appleby, JA. Mench, A. Olsson, \& BO. Hughes (Eds.), Animal Welfare (pp. 27 - 43). CABI Publishing.

18. Hewson, C.J. What is animal welfare? Common definitions and their practical consequences. The Canadian veterinary journal: La revue veterinaire canadienne 2003, 44, 496-499.

19. Zoological information management system. Available online: www.zims.Species360.org (accessed 26th December 2020).

20. Whittaker, A.L.; Barker, T.H. A consideration of the role of biology and test design as confounding factors in judgement bias tests. Applied Animal Behaviour Science 2020, 232, 105126.

21. Hill, S.; Broom, D. Measuring zoo animal welfare: Theory and practice. Zoo Biology 2009, 28, 531-544.

22. Watters, J.V.; Krebs, B.L.; Eschmann, C.L. Assessing Animal Welfare with Behavior: Onward with Caution. Journal of Zoological and Botanical Gardens 2021, 2, 75-87. 
23.

Rochlitz, I. 1997. The welfare of cats kept in confined environments. Ph.D. Thesis. Cambridge: University of Cambridge Brambell, R., Report of the Technical Committee to Enquire Into the Welfare of Animals Kept Under Intensive Livestock Husbandry Systems, Cmd. (Great Britain. Parliament), H.M. Stationery Office, pp. 1-84.

Morton, R.; Hebart, M.L.; Ankeny, R.A.; Whittaker, A.L. Assessing the uniformity in australian animal protection law: A statutory comparison. Animals 2021, 11, 35.

Mellor, D.J. Operational details of the five domains model and its key applications to the assessment and management of animal welfare. Animals 2017, 7, 60.

McCulloch, S.P. A critique of fawc's five freedoms as a framework for the analysis of animal welfare. Journal of Agricultural and Environmental Ethics 2013, 26, 959-975.

Mellor, D.; Beausoleil, N.; Littlewood, K.; McLean, A.; McGreevy, P.; Jones, B.; Wilkins, C. The 2020 five domains model: Including human-animal interactions in assessments of animal welfare. Animals 2020, 10.

Health, E.P.o.A.; Welfare. Statement on the use of animal-based measures to assess the welfare of animals. EFSA Journal 2012, 10, 2767.

Temple, D.; Courboulay, V.; Velarde, A.; Dalmau, A.; Manteca, X. The welfare of growing pigs in five different production systems in france and spain: Assessment of health. Animal Welfare 2012, 21.

Mononen, J.; Møller, S.H.; Hansen, S.W.; Hovland, A.; Koistinen, T.; Lidfors, L.; Malmkvist, J.; Vinke, C.; Ahola, L. The development of on-farm welfare assessment protocols for foxes and mink: The welfur project. Animal Welfare 2012, $21,363$. Buijs, S.; Ampe, B.; Tuyttens, F. Sensitivity of the welfare quality ${ }^{\circledR}$ broiler chicken protocol to differences between intensively reared indoor flocks: Which factors explain overall classification? Animal 2017, 11, 244-253.

Heath, C.; Browne, W.; Mullan, S.; Main, D. Navigating the iceberg: Reducing the number of parameters within the welfare quality ${ }^{\circledR}$ assessment protocol for dairy cows. Animal 2014, 8, 1978-1986.

Welfare Quality ${ }^{\circledR}$. Welfare quality ${ }^{\circledR}$ assessment protocol for poultry (broilers, laying hens); Lelystad, Netherlands, 2009.

Botreau, R.; Veissier, I.; Butterworth, A.; Bracke, M.B.M.; Keeling, L.J. Definition of criteria for overall assessment of animal welfare. Animal Welfare 2007, 16, 225-228.

Clegg, I.; Borger-Turner, J.; Eskelinen, H. C-well: The development of a welfare assessment index for captive bottlenose dolphins (tursiops truncatus). Animal Welfare 2015, 24, 267-282.

Salas, M.; Manteca, X.; Abáigar, T.; Delclaux, M.; Enseñat, C.; Martínez-Nevado, E.; Quevedo, M.; Fernández-Bellon, H. Using farm animal welfare protocols as a base to assess the welfare of wild animals in captivity - case study: Dorcas gazelles (gazella dorcas). Animals 2018, 8, 111.

Wemelsfelder, F.; Hunter, T.; Mendl, M.; Lawrence, A. Assessing the "whole animal": A free-choice-profiling approach. Animal Behaviour 2001, 62, 209-220.

9. Minero, M.; Dalla Costa, E.; Dai, F.; Murray, L.A.M.; Canali, E.; Wemelsfelder, F. Use of qualitative behaviour assessment as an indicator of welfare in donkeys. Applied Animal Behaviour Science 2016, 174, 147-153.

40. Andreasen, S.N.; Wemelsfelder, F.; Sandøe, P.; Forkman, B. The correlation of qualitative behavior assessments with welfare quality ${ }^{\circledR}$ protocol outcomes in on-farm welfare assessment of dairy cattle. Applied Animal Behaviour Science 2013, 143, 9-17.

1. Yon, L.; Williams, E.; Harvey, N.D.; Asher, L. Development of a behavioural welfare assessment tool for routine use with captive elephants. PLoS ONE 2019, 14, e0210783.

2. Miller, L.J.; Vicino, G.A.; Sheftel, J.; Lauderdale, L.K. Behavioral diversity as a potential indicator of positive animal welfare. Animals 2020, 10, 1211.

3. Shannon, C.E. A mathematical theory of communication. The Bell System Technical Journal 1948, 27, 379-423.

Howell, C.P.; Cheyne, S.M. Complexities of using wild versus captive activity budget comparisons for assessing captive primate welfare. Journal of Applied Animal Welfare Science 2019, 22, 78-96.

5. Veasey, J. Concepts in the care and welfare of captive elephants. International Zoo Yearbook 2006, 40, 63-79. 
46. Cronin, K.A.; Ross, S.R. Technical contribution: A cautionary note on the use of behavioural diversity (h-index) in animal welfare science. Animal Welfare 2019, 28, 157-164.

47. Miller, L.J.; Pisacane, C.; Vicino, G.A. Relationship between behavioural diversity and faecal glucocorticoid metabolites: A case study with cheetahs (acinonyx jubatus). Animal Welfare 2016, 25, 325-329.

48. Delfour, F.; Vaicekauskaite, R.; Garcia Parraga, D.; Pilenga, C.; Serres, A.; Isabelle, B.; Pascaud, A.; Perlado-Campos, E.; Sánchez Contreras, G.; Baumgartner, K., et al. Behavioural diversity study in bottlenose dolphin (tursiops truncatus) groups and its implications for welfare assessments. Animals 2021, 11, 1715.

49. Greggor, A.L.; Vicino, G.A.; Swaisgood, R.R.; Fidgett, A.; Brenner, D.; Kinney, M.E.; Farabaugh, S.; Masuda, B.; Lamberski, N. Animal welfare in conservation breeding: Applications and challenges. Frontiers in Veterinary Science 2018, 5, 323.

50. Rioja-Lang, F.C.; Connor, M.; Bacon, H.; Dwyer, C.M. Determining a welfare prioritization for horses using a delphi method. Animals 2020, 10, 647.

51. Mukherjee, N.; Hugé, J.; Sutherland, W.J.; McNeill, J.; Van Opstal, M.; Dahdouh-Guebas, F.; Koedam, N. The delphi technique in ecology and biological conservation: Applications and guidelines. Methods in Ecology and Evolution 2015, 6, 1097-1109.

52. Veasey, J.S. Assessing the psychological priorities for optimising captive asian elephant (elephas maximus) welfare. Animals 2020, 10, 39 .

53. Veasey, J.S. Can zoos ever be big enough for large wild animals? A review using an expert panel assessment of the psychological priorities of the amur tiger (panthera tigris altaica) as a model species. Animals 2020, 10.

54. Whittaker, A.L.; Golder-Dewar, B.; Triggs, J.L.; Sherwen, S.L.; McLelland, D.J. Identification of animal-based welfare indicators in captive reptiles: A delphi consultation survey. Animals 2021, 11.

55. Wigham, E.E.; Butterworth, A.; Wotton, S. Assessing cattle welfare at slaughter - why is it important and what challenges are faced? Meat Science 2018, 145, 171-177.

56. Raw, Z.; Rodrigues, J.B.; Rickards, K.; Ryding, J.; Norris, S.L.; Judge, A.; Kubasiewicz, L.M.; Watson, T.L.; Little, H.; Hart, B., et al. Equid assessment, research and scoping (ears): The development and implementation of a new equid welfare assessment and monitoring tool. Animals 2020, 10.

57. Fraser, D. Assessing animal welfare: Different philosophies, different scientific approaches. Zoo Biol 2009, $28,507-518$.

58. Wielebnowski, N.C. Behavioral differences as predictors of breeding status in captive cheetahs. Zoo Biology 1999, 18, 335349.

59. Wielebnowski, N.C.; Fletchall, N.; Carlstead, K.; Busso, J.M.; Brown, J.L. Noninvasive assessment of adrenal activity associated with husbandry and behavioral factors in the north american clouded leopard population. Zoo Biology 2002, 21, 77-98.

60. Brouwers, S.; Duchateau, M.J. Feasibility and validity of the animal welfare assessment grid to monitor the welfare of zoohoused gorillas gorilla gorilla gorilla. Journal of Zoo and Aquarium Research 2021, 9, 208-217.

61. Wemelsfelder, F.; Hunter, E.A.; Mendl, M.T.; Lawrence, A.B. The spontaneous qualitative assessment of behavioural expressions in pigs: First explorations of a novel methodology for integrative animal welfare measurement. Applied Animal Behaviour Science 2000, 67, 193-215.

62. Wemelsfelder, F.; Lawrence, A.B. Qualitative assessment of animal behaviour as an on-farm welfare-monitoring tool. Acta Agriculturae Scandinavica, Section A - Animal Science 2001, 51, 21-25.

63. Wolfensohn, S.; Sharpe, S.; Hall, I.; Lawrence, S.; Kitchen, S.; Dennis, M. Refinement of welfare through development of a quantitative system for assessment of lifetime experience. Animal Welfare 2015, 24.

64. Justice, W.S.M.; O'Brien, M.F.; Szyszka, O.; Shotton, J.; Gilmour, J.E.M.; Riordan, P.; Wolfensohn, S. Adaptation of the animal welfare assessment grid (awag) for monitoring animal welfare in zoological collections. Veterianry Record 2017, $181,143$.

65. Mason, G.J.; Mendl, M. Why is there no simple way of measuring animal welfare? Animal Welfare 1993, 2, 301 
66. Fernandez, E.J.; Yoakum, E.; Andrews, N. Seasonal and daily activity of two zoo-housed grizzly bears (ursus arctos horribilis). Journal of Zoological and Botanical Gardens 2020, 1, 1-12.

67. Gronqvist, G.; Kingston-Jones, M.; May, A.; Lehmann, J. The effects of three types of environmental enrichment on the behaviour of captive javan gibbons (hylobates moloch). Applied Animal Behaviour Science 2013, 147, $214-223$.

68. Swaisgood, R.R.; White, A.M.; Zhou, X.; Zhang, H.; Zhang, G.; Wei, R.; Hare, V.J.; Tepper, E.M.; Lindburg, D.G. A quantitative assessment of the efficacy of an environmental enrichment programme for giant pandas. Animal Behaviour 2001, 61, 447-457.

69. Docking, C.M.; Van de Weerd, H.A.; Day, J.E.L.; Edwards, S.A. The influence of age on the use of potential enrichment objects and synchronisation of behaviour of pigs. Applied Animal Behaviour Science 2008, 110, 244-257.

70. Videan, E.N.; Fritz, J.; Schwandt, M.L.; Smith, H.F.; Howell, S. Controllability in environmental enrichment for captive chimpanzees (pan troglodytes). Journal of Applied Animal Welfare Science 2005, 8, 117-130.

71. Kastelein, R.A.; Jennings, N.; Postma, J. Feeding enrichment methods for pacific walrus calves. Zoo Biology 2007, 26, 175-186.

72. Eskelinen, H.; Winship, K.; Borger-Turner, J. Sex, age, and individual differences in bottlenose dolphins (tursiops truncatus) in response to environmental enrichment. Animal Behavior and Cognition 2015, 2, 241-253.

73. Claxton, A.M. The potential of the human-animal relationship as an environmental enrichment for the welfare of zoohoused animals. Applied Animal Behaviour Science 2011, 133, 1-10.

74. Ward, S.J.; Melfi, V. The implications of husbandry training on zoo animal response rates. Applied Animal Behaviour Science 2013, 147, 179-185.

75. Bateson, M.; Nettle, D. Development of a cognitive bias methodology for measuring low mood in chimpanzees. PeerJ 2015, 3, e998.

76. Keen, H.A.; Nelson, O.L.; Robbins, C.T.; Evans, M.; Shepherdson, D.J.; Newberry, R.C. Validation of a novel cognitive bias task based on difference in quantity of reinforcement for assessing environmental enrichment. Animal cognition 2014, 17, 529-541.

77. Marzouki, Y.; Gullstrand, J.; Goujon, A.; Fagot, J. Baboons' response speed is biased by their moods. PloS one 2014, 9, e102562.

78. Greco, B.J.; Meehan, C.L.; Miller, L.J.; Shepherdson, D.J.; Morfeld, K.A.; Andrews, J.; Baker, A.M.; Carlstead, K.; Mench, J.A. Elephant management in north american zoos: Environmental enrichment, feeding, exercise, and training. PloS one 2016, 11, $\mathrm{e} 0152490$.

79. Mellor, D.J.; Beausoleil, N.J.; Littlewood, K.E.; McLean, A.N.; McGreevy, P.D.; Jones, B.; Wilkins, C. The 2020 five domains model: Including human-animal interactions in assessments of animal welfare. Animals 2020, 10, 1870.

80. Posta, B.; Huber, R.; Moore III, D.E. The effects of housing on zoo elephant behavior: A quantitative case study of diurnal and seasonal variation. International Journal of Comparative Psychology 2013, 26.

81. Lewton, J.; Rose, P.E. Evaluating the social structure of captive rothschild's giraffes (giraffa camelopardalis rothschildi): Relevance to animal management and animal welfare. Journal of Applied Animal Welfare Science 2020, 23, 178-192.

82. Collins, C.; Corkery, I.; Haigh, A.; McKeown, S.; Quirke, T.; O'Riordan, R. The effects of environmental and visitor variables on the behavior of free-ranging ring-tailed lemurs (lemur catta) in captivity. Zoo Biology 2017, 36, 250-260.

83. Ross, S.R.; Wagner, K.E.; Schapiro, S.J.; Hau, J.; Lukas, K.E. Transfer and acclimatization effects on the behavior of two species of african great ape (pan troglodytes and gorilla gorilla gorilla) moved to a novel and naturalistic zoo environment. International Journal of Primatology 2011, 32, 99-117.

84. Zerbe, P.; Clauss, M.; Codron, D.; Bingaman Lackey, L.; Rensch, E.; Streich, J.W.; Hatt, J.M.; Müller, D.W. Reproductive seasonality in captive wild ruminants: Implications for biogeographical adaptation, photoperiodic control, and life history. Biological Reviews 2012, 87, 965-990.

85. Botreau, R.; Bonde, M.; Butterworth, A.; Perny, P.; Bracke, M.B.; Capdeville, J.; Veissier, I. Aggregation of measures to produce an overall assessment of animal welfare. Part 1: A review of existing methods. Animal 2007, 1, 1179-1187. 